\title{
Cardiac transplantation in 150 patients at Stanford University
}

\author{
STUART W JAMIESON, EDWARD B STINSON, NORMAN E SHUMWAY
}

British Medical fournal, 1979, 1, 93-95

\section{Summary and conclusions}

A total of 150 patients underwent cardiac transplantation at Stanford between January 1968 and August 1978. Sixtytwo patients were alive at the end of this period, when the expected one-year survival rate was $70 \%$, with an attrition rate of roughly $5 \%$ thereafter. Changes in postoperative management resulting from continuing research contributed to improved survival rates. The results are equal or superior to those for recipients of renal transplants from unrelated donors.

Major centres that have suitably trained staff and appropriate facilities for cardiac surgery should perhaps reconsider cardiac transplantation as an alternative to death in certain patients.

\section{Introduction}

Orthotopic cardiac transplantation was first successfully performed experimentally in $1959 . .^{1}$ Clinical experience with cardiac transplantation began in January 1964, when Hardy et al performed the first human transplant in a patient who was in terminal cardiogenic shock. ${ }^{2}$ No human donor was available and the heart of a chimpanzee weighing $43.5 \mathrm{~kg}$ was used. Satis-

\footnotetext{
Department of Cardiovascular Surgery, Stanford University Medical Centre, Stanford, California 94305, USA

STUART W JAMIESON, MB, FRCS, British Heart Foundation-American Heart Association fellow

EDWARD B STINSON, MD, associate professor

NORMAN E SHUMWAY, MD, professor and chairman of department
}

factory cardiac output could not be achieved and attempts at resuscitation were abandoned one hour after cardiopulmonary bypass had been discontinued. Cardiac transplantation with a human donor was performed in December 1967 by Barnard, ${ }^{3}$ and this heralded a virtual epidemic of cardiac transplants throughout the world, with 100 being performed during the next 12 months by 64 teams in 22 countries. Not surprisingly, the results were poor, and after two years clinical cardiac transplantation fell into disrepute, as many began to appreciate the magnitude of the problems of rejection and infection associated with this procedure.

Although successful experimental cardiac transplantation was first achieved at Stanford University in 1959, an active research programme was continued until clinical transplantation was introduced in January 1968 with the knowledge and skill gained by a decade of experiment. The number of cardiac transplants performed at Stanford has steadily increased to roughly 25 a year. Maintaining a concurrent, aggressive research programme has allowed continuing improvements in postoperative management to be made, so that the expected oneyear survival rate is $70 \%$. This success rate is comparable or superior to those reported for renal transplantation from unrelated donors. ${ }^{4}$

We review briefly the experience in the first 150 human recipients, with special attention to modifications that have been made in clinical management since the programme began.

\section{Cardiac donors}

There were 157 donors (seven patients underwent a second transplantation), whose ages ranged from 12 to 51 years. Most cardiac donors (93) had sustained irreversible and total cerebral damage as a result of closed head injury. The concept of brain death was recognised by legislative action in California in September 1974, and the heart is removed while still beating. Other conditions in cardiac donors included cerebrovascular accidents (37) and gun-shot wounds (19). Miscellaneous causes (8) accounted for the remainder. 
The state of the heart in potential donors is carefully assessed from the medical history and by physical examination. Trauma that may have affected the heart eliminates a cardiac donor, as does a history of heart disease. Coronary arteriography and left ventriculography are performed in men older than 35 years and in women over 40 . Minor coronary artery disease is not necessarily a contraindication to the use of the heart. Recipients are matched for weight, ABO blood group, and the absence of cytotoxic effect of recipient serum on donor lymphocytes. Histocompatibility (HLA) typing is performed in all cases, but is not used prospectively for donor-recipient matching.

Donors may be brought to Stanford by ambulance or aeroplane, but in 15 recent cases the heart was removed elsewhere (up to 450 miles away) and transported in saline at $4^{\circ} \mathrm{C}$ to the operating room at Stanford where the recipient had been prepared. Ischaemic times slightly in excess of three hours have been tolerated without apparent ill effects.

\section{Cardiac recipients}

Roughly 20 patients a month are referred to Stanford as potential transplant candidates. This number undoubtedly reflects only a fraction of those with end-stage heart disease that is not amenable to any other form of treatment. Only $15 \%$ of referred patients are selected as suitable candidates for transplantation, using the following criteria:

(1) Advanced heart disease that cannot be remedied by any other form of medical or surgical treatment. Studies to exclude conditions that may be managed by other forms of treatment include cardiac catheterisation, angiocardiography, and coronary arteriography. Endomyocardial biopsy is performed in selected cases.

(2) Psychosocial stability. Psychosocial evaluation is thorough. Stability of purpose and adequate emotional support is necessary for postoperative rehabilitation and the outpatient co-operation that is required for close, long-term medical supervision.

Potential recipients are eliminated if they have a systemic disorder that would limit recovery or survival, and an age limit of 55 years is set on similar grounds. Diabetes mellitus that requires insulin treatment or any active infection are also contraindications. Unresolved pulmonary infarction (since this predisposes to postoperative pulmonary abscess) and a pulmonary vascular resistance greater than 8 Wood units $^{5}$ (because the normal donor right ventricle cannot acutely match such an external work load) are also contraindications. Roughly $60 \%$ of recipients have end-stage coronary artery disease and 30\% idiopathic cardiomyopathy.

\section{OPERATION}

The technique of cardiac transplantation has been fully described. ${ }^{6}$ Cardiopulmonary bypass is instituted centrally as for other cardiac operations, then, leaving the posterior walls of the recipient atria in situ, the heart is excised. The donor heart is sewn in, starting with the atria, and then the aorta and pulmonary artery anastomoses are constructed.

\section{EARLY POSTOPERATIVE CARE}

Immediate postoperative care is similar to that after routine cardiac surgery, except that most patients receive temporary inotropic support with isoprenaline because of temporary depression of donor cardiac output, and immunosuppression is instituted. Scrupulous attention is paid to reverse isolation techniques. The patients remain in the intensive care unit in isolated cubicles for an average of one month, and in the general ward (in private rooms) for a further month. The average stay in hospital is 55 days.

\section{IMMUNOSUPPRESSION}

Indefinite immunosuppression is required, although some form of adaptation occurs, with patients showing a progressive reduction in the frequency of rejection crises with time. Immediately after transplantation a "triple" protocol incorporating corticosteroids, azathioprine, and antihuman thymocyte globulin (ATG) produced in rabbits is used. The latter is used routinely only for two weeks after transplantation, but is reinstituted for rejection crises.

\section{REJECTION}

Rejection episodes are diagnosed on the basis of clinical examination (diastolic gallop), electrocardiographic appearances (decrease in QRS voltage, atrial arrhythmias, occasional depression of ST segments, shift of ECG axis), and endomyocardial biopsy, which is performed weekly for two months or if rejection is suspected. Only $10 \%$ of patients do not show symptoms of acute rejection after transplantation, and early detection and rapid reversal of rejection is essential for survival.

Immunological monitoring is carried out by measuring circulating T ("killer") lymphocytes by the spontaneous rosette-formation test with sheep erythrocytes. This test, however, is most useful during the first 30 days, after which there is an increase in false-positive results. The dosage and frequency of ATG administration are guided by measuring the half life of circulating rabbit globulin and the rosette test. The primary goal in the first 30 days after transplantation is to depress the number of $\mathrm{T}$ cells (as measured by the rosette test) to less than $10 \%$ of normal. A rise in the rosette fraction is seen one to three days before rejection is confirmed by biopsy.

Acute rejection episodes confirmed histologically are treated with increased immunosuppression. "Pulses" of methylprednisolone (one gram intravenously daily for three days) are administered as well as small doses of actinomycin D. A short course of ATG is begun again. Heparin is given to avoid possible vascular damage by fibrin and platelets. Of rejection episodes treated in this way, $95 \%$ can be reversed. Late postoperative rejection episodes are less severe and frequent. They are usually treated by increasing the dose of oral prednisone.

\section{LATE MANAGEMENT}

Patients are seen in the outpatient clinic twice weekly for two months, weekly for two months, then monthly if still resident in the area. Chest $x$-ray examination, electrocardiography, and appropriate laboratory investigations are performed at these times. Once a year patients are readmitted for coronary arteriography, left ventriculography, and cardiac biopsy. Patients are maintained on a low cholesterol, low sodium diet. Antiplatelet agents are used to control the development and progression of the atheroma-like condition that develops after most organ grafts. (The development of ischaemia in the cardiac graft is painless since the heart is denervated.) Rejection episodes diagnosed electrocardiographically and by cardiac biopsy are treated aggressively.

Retransplantation has been used to treat both advanced atherosclerosis in the graft and intractable acute rejection. In these cases it is the only alternative to certain death and seven patients have undergone a second transplantation (three successfully).

\section{Results}

The figure shows the survival rate in the 150 transplant recipients, together with the survival rate of patients who were accepted as recipients, but died before a suitable donor was found. The one-year

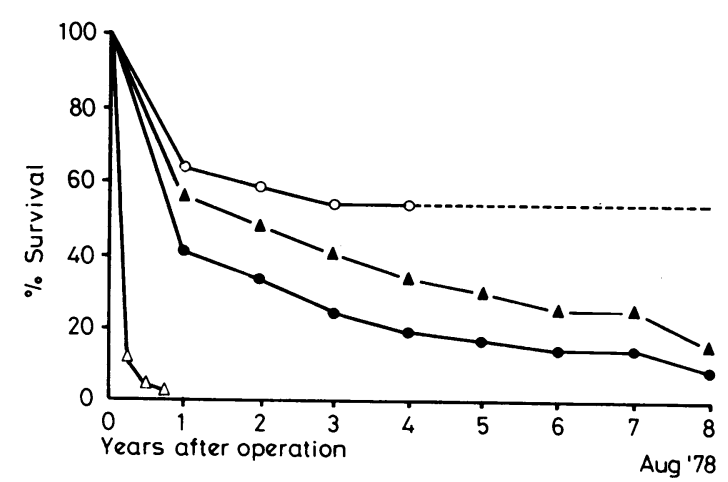

Survival rate in 150 patients who underwent cardiac transplantation $(\Delta)$ and in patients who did not undergo transplantation because suitable donors were not found $(\triangle)$. = Patients who underwent transplantation during January 1968-September 1973; $O=$ patients who underwent transplantation during October 1973-August 1978. 
survival rate has improved from $22^{\circ}{ }_{0}$ in 1968 to $68^{\circ}{ }_{o}$ in 1977 . Survival rates of patients who underwent transplantation in the last five years have improved (figure). Apart from increased experience, the enhanced survival correlates with several distinct improvements in management that have been developed or tested in the laboratory before clinical use. The endomyocardial bioptome provides histological evidence of rejection and allows myocardial histology to be correlated with drug treatment. The use of antithymocyte globulin produced in rabbits was started in October 1973 and has been used in the last 86 patients. Since the introduction of rabbit ATG, measurement of the half life of circulating rabbit globulin has been used to modulate immunosuppressive treatment in individual patients, and, as described above, early activation of the immune response has been detected by measuring circulating $\mathrm{T}$ lymphocyte concentrations.

Since 1970 an acute awareness of graft atherosclerosis has prompted the treatment of all patients with dipyridamole and warfarin, and this regimen, together with the reduction of plasma lipid concentrations associated with a low-fat diet, may be responsible for the observed reduction of graft atherosclerosis as measured by yearly coronary arteriograms. Two patients with advanced immune-mediated athersclerosis, however, needed to undergo a second transplantation.

The complications of generalised immunosuppression are the major factors accounting for morbidity and mortality after cardiac transplantation. Infection is the most serious complication, though osteoporosis, aseptic necrosis of the joints, and change in appearance may affect rehabilitation. Infection caused 45 deaths $(30 \%$ of all patients), the lungs being the most common site. Out of 319 infectious episodes, $150\left(47^{\circ}{ }_{0}\right)$ were pulmonary, $35\left(11^{\circ}{ }_{0}\right)$ septicaemic, $22(7 \%)$ occurred in the urinary tract, and $10(3 \%)$ in the central nervous system. Infection is more common after cardiac transplantation than renal transplantation because more intense immunosuppression is needed to maintain cardiac function during rejection. The care of cardiac transplant recipients is thus dissimilar to that of recipients of hepatic and renal grafts because of the narrower margin between maintaining effective organ function and death, and at Stanford the care of transplant recipients is solely in the hands of the cardiac surgeon until the patient is discharged. Rejection accounted for 19 deaths $\left(13^{\circ}\right.$ of all patients).

\section{Discussion}

For the patient in end-stage cardiac failure that is unresponsive to maximal medical and surgical treatment there is a choice between cardiac transplantation and death. For patients who fulfil the criteria for suitable cardiac recipients the one-year survival rate is close to $70^{\circ}{ }^{\circ}$. In $90^{\circ}$, of long-term survivors in our series physical activity has returned to normal and most have resumed active employment.

There are few published data on the cost of cardiac transplantation. In the United States the cost of transplantation has been estimated at roughly $\$ 40000$ for the transplant year and $\$ 2500$ yearly thereafter. The cost to society of an occupationally incapacitated person would be $\$ 32000$ in support money and loss of occupational contributions. Thus expenditure on cardiac transplantation would roughly equal the cost of medical treatment for end-stage cardiac disease, even if only $30 \%$ of transplant recipients were rehabilitated. ${ }^{7}$ Naturally, an economic value cannot be placed on life or rehabilitation for the person or family concerned.

Factors that contribute to the relative success of transplantation at Stanford are a concomitant research programme that has led to the development and the continued refinement of various aspects of patient care and management of rejection, the total commitment of a section of the cardiovascular surgery team and research workers to the transplant programme, and an increased awareness of the unique problems associated with cardiac transplantation that derives from 10 years of experience in this field.

Other centres that have the appropriate facilities for cardiac transplantation and suitably trained staff should perhaps reconsider cardiac transplantation as an alternative to death in certain patients.

This work was partly supported by a grant (HL13108) from the National Heart, Blood and Lung Institute of the National Institute of Health.

\section{References}

${ }^{1}$ Lower, R R, and Shumway, N E, Surgical Forum, 1960, 11, 18

2 Hardy, J D, et al, fournal of the American Medical Association, 1964, 188, 1132.

${ }^{3}$ Barnard, C N, South African Medical fournal, 1967, 41, 1257.

${ }^{4}$ Human Renal Transplant Registry, Transplant Proceedings, 1977, 9, 9.

5 Wood, P, Diseases of the Heart and Circulation, 2nd edn. London, Eyre and Spottiswoode, 1956

${ }^{6}$ Stinson, E B, et al, American fournal of Surgery, 1969, 118, 182.

7 Thomas, F T, and Lower, R R, Surgical Clinics of North America, 1978, 58, 335.

(Accepted 26 October 1978)
GPs have had notice from the DHSS that the ten-dose container of oral polio vaccine may lose its potency after it has been opened. Is it reasonable to give polio at the same time as smallpox to people going abroad, and to store the smallpox in the freezer part and the oral polio in the main part of the refrigerator, and to throw both smallpox and polio phials away at the same time? (The smallpox usually lasts 14 days.)

The DHSS official advice is that live viral vaccines should not be administered at the same time but that a three-week interval should be allowed between them. The American practice, however, is to give several live viral vaccines at the same time as it is felt in that country that there is no risk in so doing. As many travellers rarely leave themselves sufficient time for proper protection and polio is quite common among recent arrivals, in cases of urgency I think your questioner's procedure a reasonable one. With regard to storage of the oral polio once it has been opened, the DHSS advise that it should be thrown away immediately, and this should be adhered to as any infection introduced into the container would destroy its potency. If the smallpox vaccine distributed in the plastic tubing by the Lister Institute is stored in the freezer compartment of the refrigerator at about $-10^{\circ} \mathrm{C}$ the potency is assured for one year.

Should a 16-year-old girl have her tonsils removed after five attacks of tonsillitis in five months?

Preservation of the tonsils in a patient over the "catarrhal age" (13-14 years) is not a first consideration. Therefore, if the patient's education or working life is being seriously disrupted by repeated attacks of tonsillitis, a tonsillectomy would appear to be the most sensible treatment. 